\title{
La innovación docente en evaluación formativa y metodología participativa: Un proyecto compartido a raíz de la implantación de los nuevos grados
}

\author{
Ma Luisa Santos Pastor*, F. Javier Castejón Oliva y L. Fernando Martínez Muñoz \\ Universidad Autónoma de Madrid
}

(Received August 16, 2011; Accepted January 28, 2012)

\begin{abstract}
RESUMEN: El artículo se centra en exponer y analizar los procesos de innovación que se han puesto en marcha durante tres cursos académicos por un grupo de profesores y profesoras universitarios que imparten docencia en la Universidad Autónoma de Madrid, en la nueva titulación de Grado en Ciencias de la Actividad Física y del Deporte. El principal objetivo es dar respuesta a los interrogantes planteados sobre las nuevas formas de enseñar, mediante metodologías participativas y sistemas de evaluación formativos. Se presentan las fases del proceso seguido, las acciones emprendidas en cada una de ellas, así como los resultados obtenidos, ventajas e inconvenientes encontrados en su desarrollo y las soluciones aportadas. Se incide en las repercusiones que ha tenido sobre la mejora de la docencia, además de la utilidad de los resultados obtenidos en otros contextos similares.
\end{abstract}

Palabras clave: innovación docente, educación superior, metodología participativa, evaluación formativa, desarrollo profesional.

Educational innovation in formative assessment and participatory methodology: following the introduction of new degrees

ABSTRACT: This paper is aimed at presenting and analysing the innovation processes implemented during three academic years by a group of teachers from the Universidad Autónoma de Madrid in the Science Degree in Physical Activity and Sport. The main objective is to provide response to the questions raised on the new ways of teaching, by means of participatory methodologies and formative assessment systems. The stages of the process followed, the actions undertaken for each of them, their outcomes, advantages and drawbacks encountered during their development, as well as the solutions provided for them are presented herein. The focus is on its impact on the improvement of teaching and the usefulness of the results for other similar contexts.

Keywords: teaching innovation, higher education, participative methodology, formative assessment, professional development.

\section{INTRODUCCIÓN}

Este artículo describe la experiencia de innovación docente colectiva que se ha desarrollado durante tres cursos académicos en la Universidad Autónoma de

\footnotetext{
*Correspondencia: Marisa Santos Pastor, Facultad de Formación de Profesorado y Educación, 
Madrid, en la nueva titulación de Grado en Ciencias de la Actividad Física y del Deporte. Nuestros propósitos se han basado en diseñar, aplicar y valorar diferentes estrategias docentes centradas en metodologías participativas y en evaluación formativa; así como compartir información para la mejora docente.

Las razones que justifican la elección de estas temáticas para el desarrollo de los proyectos de innovación son:

1. Adecuar nuestra acción docente a los planteamientos metodológicos y de evaluación pautados por el Espacio Europeo de Educación Superior (EEES). Particularmente frente a estos dos interrogantes: (a) ¿Cómo se puede llevar a cabo la evaluación formativa en las circunstancias particulares de una universidad, pero que pueda servir como ejemplo o acicate para la comunidad universitaria? (b) ¿Cómo pueden desarrollarse en la práctica metodologías participativas?

2. Renovar la metodología y la metodología centradas ambas en el aprendizaje del alumnado, solicitando que sea un agente activo y autónomo en ese proceso.

3. Analizar y compartir las experiencias individuales del aula, buscando soluciones acordes a nuestros planteamientos. El auténtico valor de este proyecto incide en las posibilidades de innovación que facilitan el desarrollo profesional de los docentes involucrados, los cuales ponen en común experiencias, incertidumbres y dudas, así como la búsqueda de soluciones.

Nos constituimos como grupo de trabajo colaborativo desde 2008, aprovechando la convocatoria anual de Proyectos para el Desarrollo de Enseñanzas para la Convergencia con Europa, financiados por el Vicerrectorado para el Desarrollo de las Enseñanzas y la Formación Continua de la Universidad Autónoma de Madrid. Desde ese año, coincidiendo con cada convocatoria, nos centramos en unos propósitos de innovación relacionados con la metodología y la evaluación. El grupo ha ido fluctuando entre ocho y diez profesores para cada convocatoria. El procedimiento de trabajo se ha llevado a cabo en reuniones periódicas donde se han debatido y reflexionado las propuestas de acción docente individuales y colectivas. En todos estos proyectos, la práctica docente se analiza, se cuestiona y se replantea para aprender colectivamente (Knight, 2005), para innovar en el aula, para incidir en el desarrollo profesional y para animar a buscar nuevos compromisos (Benito, Cruz, e Icarán, 2005; Margalef y Álvarez, 2005).

La selección de esta temática está condicionada por el hecho de que varios docentes participantes forman parte de la Red de Evaluación Formativa y Compartida en Docencia Universitaria. Su actividad principal se basa en el desarrollo de experiencias de innovación, centradas en el uso de sistemas de evaluación formativa y compartida así como metodologías participativas, orientadas al mayor desarrollo de competencias profesionales, en la línea del modelo ECTS (European Credit Transfer and Accumulation System) (López-Pastor, 2011). La meto- 
dología de trabajo en la Red está basada en la realización de sucesivos ciclos de investigación-acción y los resultados han demostrado un conjunto de aspectos positivos en su aplicación, pero también dificultades a las que se sigue tratando de buscar soluciones (Buscá, Pintor, Martínez, y Peire, 2010; López-Pastor, 1999, 2009; Santos, Martínez, y López-Pastor, 2009).

La innovación no sólo busca hacer cosas distintas, sino hacerlas mejor manteniendo los cambios en el tiempo (Zabalza, 2003-2004). Este proceso requiere de ciertas actitudes y capacidades por parte del profesorado, entre las que destacamos: apertura, flexibilidad para adaptarse a los cambios y hacer reajustes necesarios de manera progresiva, actualización de conocimientos docentes y recursos disponibles para articular nuevos métodos docentes y de evaluación formativa, evitando caer en la improvisación, así como la mejora de las prácticas docentes que busca generar cambios viables y útiles, que implique a los protagonistas (alumnado y profesorado) y a la institución (departamento, titulación, facultad y universidad) (Hannan y Silver, 2006; Zabalza, 2007). En suma, un conjunto de características personales y profesionales que requieren una implicación permanente y un compromiso individual que trata de mejorar el desarrollo profesional docente. Además de las características ya señaladas, se aborda el proceso de innovación contando con unas condiciones iniciales, que ya han sido tratadas por otros autores (Zabalza, 2003-2004): la creación de un seminario permanente formado por el grupo docente implicado y la facilitación institucional con ayudas y apoyos a la innovación.

En cuanto a metodología, el fundamento de nuestra innovación se basa en: (a) Adquirir un mayor compromiso como docentes, acercarnos al alumnado y romper con lo tradicional (Martínez, Santos, y Sicilia, 2006). (b) Diseñar propuestas metodológicas coherentes con prácticas de evaluación democráticas, impulsoras del protagonismo y responsabilidad del alumnado, fundamentadas en el aprendizaje dialógico y no sólo orientadas por la calificación (López-Pastor, 2009; Martínez et al., 2006; Santos et al., 2010; Santos, Castejón, Martínez, y Muñoz, 2011). En relación con la evaluación formativa es necesario no sólo innovar mediante nuevos instrumentos de evaluación, sustitutos o complementarios al examen, sino que se requiere: (a) Situar al estudiante como protagonista de su proceso de aprendizaje. (b) Generar un proceso que oriente todos los medios al servicio de un aprendizaje valioso y profundo (autónomo, significativo y útil) (Entwistle, 1990). En definitiva, una evaluación al servicio del aprendizaje y como referente de la calidad del profesorado (Biggs, 2006). La evaluación continua y formativa es avalada por una revisión del proceso de aprendizaje del alumnado, lo que permite que haya una mejora del mismo, siendo un aspecto central de nuestra acción formativa.

\section{OBJETIVOS DE LA EXPERIENCIA DE INNOVACIÓN}

Los objetivos de la experiencia de innovación sobre metodologías participativas y evaluación formativa se pueden sintetizar en: 
1. Comprobar las posibilidades pedagógicas, ventajas e inconvenientes de la evaluación formativa en la enseñanza universitaria, así como la relación que guarda con la metodología utilizada.

2. Planificar, diseñar y poner en práctica herramientas que permitan desarrollar metodologías activas y sistemas de evaluación formativa.

3. Analizar la influencia que tiene el desarrollo de este tipo de instrumentos y herramientas de evaluación en el incremento del aprendizaje del alumnado universitario y en la mejora de su rendimiento académico.

\section{PROCEDIMIENTO GENERAL DE ACTUACIÓN}

El trabajo desarrollado se divide en varias fases (ver tabla 1), en forma de ciclos de investigación-acción, coincidentes con las convocatorias anuales de proyectos que se van adjudicando.

Tabla 1. Fases del Proyecto de Innovación Sobre Metodología y Evaluación

\begin{tabular}{|c|c|c|c|}
\hline Año & Título del Proyecto & Temáticas & Actividad \\
\hline $\begin{array}{c}2009 \\
\text { Fase } 1\end{array}$ & $\begin{array}{l}\text { Análisis de relaciones entre } \\
\text { evaluación formativa, } \\
\text { metodologías activas, carga } \\
\text { de trabajo y rendimiento } \\
\text { académico en la implantación } \\
\text { anticipada del modelo ECTS. }\end{array}$ & $\begin{array}{l}\text { Elaboración de un } \\
\text { marco de actuación } \\
\text { docente sobre modelos } \\
\text { de evaluación } \\
\text { formativa y } \\
\text { participativa. }\end{array}$ & $\begin{array}{l}\text { Puesta en práctica de } \\
\text { modelos de evaluación } \\
\text { formativa y su relación } \\
\text { con metodologías } \\
\text { activas, así como la } \\
\text { repercusión sobre el } \\
\text { aprendizaje del } \\
\text { estudiante. }\end{array}$ \\
\hline $\begin{array}{c}2010 \\
\text { Fase } 2\end{array}$ & $\begin{array}{l}\text { El portafolio digital como } \\
\text { instrumento de evaluación } \\
\text { formativa en la titulación de } \\
\text { Grado de Ciencias de la } \\
\text { Actividad Física y el Deporte. }\end{array}$ & $\begin{array}{l}\text { El portafolio como } \\
\text { instrumento de } \\
\text { evaluación formativa. }\end{array}$ & $\begin{array}{l}\text { Diseñar y aplicar el } \\
\text { portafolio digital como } \\
\text { herramienta que nos } \\
\text { posibilita desarrollar } \\
\text { estrategias de } \\
\text { evaluación formativa } \\
\text { y compartida. }\end{array}$ \\
\hline $\begin{array}{c}2011 \\
\text { Fase } 3\end{array}$ & $\begin{array}{l}\text { Los wikis como recurso } \\
\text { metodológico para el } \\
\text { aprendizaje colaborativo en } \\
\text { los estudios de Grado de } \\
\text { CAFD. }\end{array}$ & $\begin{array}{l}\text { Los wikis como } \\
\text { oportunidad para el } \\
\text { trabajo colaborativo y } \\
\text { una evaluación } \\
\text { compartida. }\end{array}$ & $\begin{array}{l}\text { Diseñar wikis como } \\
\text { herramienta que nos } \\
\text { posibilita desarrollar } \\
\text { evaluación formativa } \\
\text { y metodologías } \\
\text { participativas. }\end{array}$ \\
\hline
\end{tabular}

Cada fase del proyecto ha tratado de profundizar sobre un aspecto de la metodología participativa y la evaluación formativa: (a) Primera, de carácter genérico, donde se desarrollaron protocolos de aplicación de evaluación formativa en las materias implicadas. (b) Segunda, se crearon instrumentos que nos permitieran gestionar dicha evaluación, siendo el portafolio digital el centro de interés de nuestra experiencia. (c) Tercera, se implementaron los wikis como herramienta de trabajo en la docencia universitaria. 
Cada una de estas fases comprende aspectos relacionados con metodologías participativas y evaluación formativa que cada docente pone en práctica con su grupo de estudiantes.

\section{PRIMERA FASE. UN MARCO DE PARTIDA PARA LA EVALUACIÓN FORMATIVA}

Por evaluación formativa entendemos aquella que está orientada al aprendizaje del alumnado, como proceso. Nos hemos centrado en generar un marco común sobre el que fundamentar y aplicar la evaluación formativa, asumiendo diferentes retos docentes (Martínez et al., 2006). Las propuestas realizadas para cada materia eran singulares, ya que cada docente tenía distintas experiencias previas en relación a la puesta en práctica de estrategias de evaluación formativa, si bien todas se enmarcan dentro de las disposiciones que tiene la universidad para el desarrollo de las enseñanzas de las diferentes titulaciones de Grado. La propuesta de innovación en evaluación fue plantear al alumnado al menos dos opciones: continua y final, seleccionada libremente tras la aceptación de los compromisos que supone cada una.

La vía de evaluación continua (base de nuestro proyecto) se consensua y negocia en el grupo, con unos criterios de evaluación públicos que orientan su aprendizaje, atendiendo a las necesidades y posibilidades detectadas, considerando el compromiso y la responsabilidad del alumnado (Santos et al., 2009). Asimismo, se diferencian dos tipos de propuestas: (a) Autoevaluación-autocalificación, donde el alumnado adquiere la responsabilidad de evaluar y calificar su proceso de formación y de aprendizaje de la materia. Se parte de un contrato inicial en el que cada estudiante se compromete a cumplir unos requisitos, tales como: asistencia, participación, elaboración de la carpeta del alumnado (lecturas, recensiones, trabajos grupales, etc.), realización del informe de autoevaluación, asistir a la asamblea final de evaluación. (b) Empleo de una escala de calificación graduada (Castejón, 2009), donde se dan tres modalidades de evaluación: (1) Continua y colaborativa. (2) Sin colaboración. (3) Sin asistencia. Cada una de ellas exige un compromiso de trabajo acorde con las posibilidades del alumnado.

Esta idea de evaluación que utilizamos se relaciona con el uso de planteamientos didácticos afines a una metodología participativa. Se basa en la selección de actividades de enseñanza-aprendizaje, tales como lecturas y recensiones de documentos, proyectos didácticos, aprendizaje basado en problemas (ABP), casos prácticos, prácticas simuladas, uso de plataformas virtuales de aprendizaje, tutorías planificadas y sistematizadas y su relación con los instrumentos de evaluación utilizados (trabajos individuales: recensiones, carpetas, portafolio, etc.; trabajos colectivos: proyecto didáctico, estudios de caso, ABP, etc.).

Los principios y pautas metodológicas y de evaluación establecidos por el grupo docente a lo largo de esta primera fase, nos permitieron analizar las ventajas y dificultades que surgieron durante la puesta en práctica de la experiencia (ver tabla 2). 


\section{Tabla 2. Ventajas y Dificultades de la Evaluación Formativa}

\section{Ventajas}

El alumnado siente que el docente está haciendo todo lo posible por ser justo, transparente y democrático. En este sentido, la evaluación formativa propicia que el alumnado reconozca la voluntad de innovación y progreso del profesorado, de tal forma que el concepto y la imagen del profesor mejoran habitualmente.

La evaluación formativa dinamiza el aprendizaje significativo y el aprender a aprender, propiciando que el estudiante se involucre y sea el protagonista del proceso de adquisición de conocimientos.

Mejora la motivación y confianza, ya que se hace responsable del proceso que lleva a cabo.

Se da la oportunidad al alumnado de opinar sobre la organización de la asignatura.

El alumnado vivencia una forma alternativa a la evaluación más tradicional, lo cual es bueno, especialmente si su futuro

profesional está relacionado con el mundo de la enseñanza. La evaluación formativa exige al profesor una constante actualización de contenidos y un proceso continuo de reflexión sobre situaciones reales.

Mejora el criterio del profesorado a la hora de seleccionar contenidos que sean coherentes, valiosos, relevantes y útiles. Se mejora la conexión entre teoría y práctica. La evaluación formativa implica una tutela que conduce a una mayor y mejor información, tanto de aspectos globales de la materia como de aspectos específicos o particulares.

\section{Dificultades}

Mayor carga de trabajo para el alumnado. Exige un esfuerzo continuado, que no siempre pueden seguir.

Proceso lento que requiere un tiempo para que el estudiante pueda comprenderlo, pues no son procesos de evaluación habituales.

Es difícil llevar a cabo con un elevado número de estudiantes por grupo. El trabajo en grupo por parte de los alumnos/as desemboca, a veces, en conflictos derivados de la implicación desequilibrada de los componentes del grupo. En otras ocasiones, no se desarrolla trabajo en grupo, por la distancia, horarios y responsabilidades laborales.

Las tutorías suponen una ampliación horaria excesiva y se pueden solapar con las clases de otras materias.

La cercanía con el alumnado provoca una proximidad afectiva que puede condicionar el proceso de valoración del alumnado.

Posibilidad o riesgo de entrar en un sistema de evaluación/calificación "blando" o poco exigente.

La necesidad de buscar soluciones a los problemas encontrados en esta fase inicial, junto con el interés por seguir profundizando sobre las posibilidades de la evaluación formativa, fueron los motivos que nos indujeron a continuar con las siguientes fases de la experiencia de innovación.

\section{SEGUNDA FASE. EL PORTAFOLIO COMO INSTRUMENTO DE EVA- LUACIÓN FORMATIVA}

En esta fase nos centramos en la utilización de nuevas formas de evaluación y metodología más coherentes, motivados por la necesidad de buscar nuevas implicaciones del alumnado en su aprendizaje. Para que haya una evaluación 
formativa es necesario construir nuevos instrumentos de evaluación, sustitutos o complementarios al tradicional examen, además de poner en práctica estrategias que permitan aprendizajes valiosos y profundos (autónomos, significativos y útiles). Por ello, en una segunda fase del proyecto de innovación, nos planteamos utilizar una herramienta que nos ayudará a gestionar, supervisar y valorar las múltiples tareas que se proponen para facilitar el aprendizaje del alumnado: el portafolio digital en la plataforma Moodle. Consideramos que su utilización supone una mejora en sus aprendizajes, porque es una herramienta contrastada para la evaluación y, particularmente, para la evaluación formativa (Arter y Spandel, 1992; González, 2007; Klenowski, 2007; Lyons, 2003; Pozo y García, 2006; Santos et al., 2011) y en el caso de los portafolios electrónicos, permiten ampliar las posibilidades del portafolio habitual (Capllonch y Castejón, 2007). Por ello, se convierte en un instrumento de evaluación y una herramienta metodológica eficaz.

El proceso de esta fase se secuenció de la siguiente manera: (a) Planificación y formación. (b) Implementación y seguimiento. (c) Finalización y evaluación.

El diseño del portafolio fue común para todos los docentes, con las adaptaciones necesarias para cada materia. Su estructura estaba compuesta por cuatro grandes apartados:

1. Introducción-presentación. Expectativas ante la asignatura. Compromisos. Justificación de la selección de los trabajos presentados. Conexión con otros conocimientos y experiencias.

2. Trabajo personal-individual. Recoge evidencias del trabajo individual.

2.1. Evidencias obligatorias. Calendario. Registro de asistencia. Registro de incidencias. Otros ((Auto) biografía personal). Lecturas, mapas conceptuales y reflexiones. Diario de aprendizaje. Sesiones prácticas. Interrogantes sin resolver.

2.2. Evidencias optativas. Ampliación de contenidos.

3. Trabajo colectivo. Se presentan las acciones de aprendizaje colaborativo. 3.1. Evidencias Obligatorias. Trabajo colaborativo de aplicación práctica.

3.2. Evidencias Optativas

4. Conclusiones. Síntesis del trabajo realizado en la asignatura. Tiempo dedicado. Propuestas de mejora. Informes de evaluación (autoevaluación, co-evaluación y heteroevaluación).

Su elaboración atendió al proceso, a través del cual se van construyendo y recopilando las evidencias de aprendizaje que deberán plasmarse en la elaboración final del mismo. Por tanto, se realizan diferentes microtareas (informes, lecturas, resoluciones de casos, reflexiones de prácticas, etc.) que son objeto de revisión por parte del docente, con el fin de ayudar al alumnado a completar y mejorar su proceso de aprendizaje. Esta retroalimentación realizada durante el proceso (feed foward), debe repercutir en la mejora de las tareas que han de incorporar al portafolio final. 
Todo este proceso era continuamente evaluado por parte del profesorado del seminario en las diferentes reuniones periódicas que tenía establecidas, con el fin de aportar respuestas ante los problemas que iban surgiendo en cada una de las materias en las que se implementaban el portafolio. Sirva como muestra una evidencia extraída de uno de los encuentros del grupo:

...Se comentaron algunas cuestiones interesantes a considerar en su aplicación, entre ellas:

- Posibilidad de construir una plantilla de portafolio para que el alumnado se dedique simplemente a utilizarlo.

- Hay que construir un protocolo-guía para el estudiante.

- Es necesario ir tomando decisiones sobre la plataforma que vamos a utilizar (me inclino por sites, más que moodle).

- Con el fin de familiarizaros con el sites sería interesante que fuéramos probando cómo funciona. Es sumamente sencillo y muy útil (Acta reunión, de 10 de junio de 2010)

A pesar de las incertidumbres, podemos afirmar que son más los beneficios que la problemática que genera su puesta en práctica para el desarrollo de una evaluación formativa (Santos et al., 2011). En esta línea de indagación continua y permanente, tratamos de ir dando respuesta a las dudas y dificultades que nos han ido surgiendo. En la tabla 3 presentamos las ventajas y obstáculos encontrados en esta fase del proyecto de innovación.

\section{Tabla 3. Ventajas y Dificultades en el Uso del Portafolio}

\section{Ventajas}

La posibilidad de seguimiento permanente y continuo del trabajo del alumnado.

La mejora en la distribución y organización de las tareas de aprendizaje y evaluación. Servir de guía y orientación para el trabajo autónomo de cada estudiante en las tareas de evaluación.

Permite una mejor organización y gestión docente para la distribución de las tareas de evaluación.

Mejora la calidad de la formación de los y las estudiantes.

\section{Dificultades}

Dificultades técnicas con el uso de la plataforma Moodle.

Buscar un diseño de portafolio adecuado y equilibrado con los requisitos de la evaluación formativa.

Incertidumbre de su puesta en práctica. Exceso de carga docente. Tiempo muy escaso para el feed-back individual. Exceso de carga de trabajo para el alumnado. Tiempo excesivo dedicado a la elaboración del portafolio.

Para finalizar este apartado, queremos destacar el interés y el beneficio que para el profesorado y alumnado ha supuesto trabajar en equipo, desarrollando procesos de reflexión compartida, haciendo más liviana la inseguridad de la propuesta de innovación y favoreciendo la puesta en práctica de una evaluación formativa. 


\section{TERCERA FASE. LOS WIKIS COMO OPORTUNIDAD PARA EL TRABAJO COLABORATIVO Y LA EVALUACIÓN COMPARTIDA}

Actualmente nos encontramos realizando una nueva fase de nuestro proyecto, que nos permitirá seguir profundizando sobre el diseño de tareas para el portafolio, en este caso, relacionado con el trabajo en grupo. Se trata de diseñar y poner en práctica los wikis como herramienta de sistemas de evaluación formativa, así como estrategias y planteamientos metodológicos potenciadores del aprendizaje colaborativo en el aula universitaria. En trabajos de diversos autores se han expuesto las posibilidades educativas de los wikis acordes con las necesidades formativas del alumnado en el marco del EEES (González y Miralbell, 2009; Lázaro, Pena y Vitalaru, 2009). Se ha demostrado que fomenta su participación e incentiva el aprendizaje entre los discentes (Anguita, García, Villagrá y Jorrín, 2009; Montenegro y Pujol, 2009; Robles et al., 2009) además de servir como espacio para el debate. Entre las ventajas de su utilización, destacan: (a) Constituir una motivación para el aprendizaje del alumnado al involucrarse en proyectos colaborativos que requieren un trabajo compartido, consensuado y equilibrado. (b) El profesorado puede estar al corriente de las aportaciones y el nivel de compromiso de cada uno y cada una de sus estudiantes, ya que puede interactuar de manera permanente con sus aportaciones. (c) Permite el trabajo compartido entre estudiantes y profesorado, distribuyendo tareas, al tiempo de servir de punto de encuentro para poner en común las reflexiones que sugieren los diferentes temas planteados.

En nuestro caso, hemos seleccionado como centro de interés el último apartado, que nos permiten generar actividades acordes a nuestros intereses, tales como la creación de ensayos científicos, trabajos de investigación, reflexiones colaborativas u otro tipo de tareas didácticas. Además, hemos tratado de que los propios docentes puedan emplear los wikis para organizar los recursos didácticos de la materia que imparten (subir presentaciones, apuntes, ejercicios, enlaces de interés, etc.) e incluir los aportados por el alumnado.

Articular de forma coherente los procesos metodológicos con los de evaluación, requiere de una correcta utilización de los wikis. En nuestro caso, lo hemos relacionado con el proceso metodológico desde un doble planteamiento: (a) El procedimiento seguido para su diseño y puesta en práctica, donde entendemos que deben aparecer las posibilidades pedagógicas del trabajo en equipo, y cómo se materializa en una documentación al alcance del grupo, para que pueda ser empleado en el desarrollo de cada una de las materias. (b) La forma de entender el aprendizaje, pues supone una apropiación por parte del alumnado de las ideas y conceptos de cada materia, que deben ser correctamente argumentadas siguiendo las pautas de la puesta en práctica de los wikis. Hemos creído que esta forma de entender una propuesta de metodología participativa redunda en su aprendizaje profundo de las materias. Asimismo, el profesorado ofrece orientaciones durante el proceso y aporta feedback en base al avance de dicho aprendizaje.

La secuenciación del trabajo desarrollado está relacionado con las activida- 
des que se proponen en el proceso de innovación, las cuales se sintetizan en la tabla 4.

Tabla 4. Fases y Actividades de la Innovación con Wikis

\section{Fases}

Planificación y

formación

Implementación y seguimiento

Finalización y evaluación

\section{Actividades}

Diseño de las acciones del proceso de innovación. Formación del profesorado sobre el uso de los wikis en Moodle (junio-septiembre).

Adaptación de wiki en Moodle a la singularidad de cada asignatura. Seguimiento de las actividades en reuniones quincenales para tratar ventajas e inconvenientes. Resolución individual y en grupo tanto didácticas como técnicas.

Elaboración de informe final. Principales resultados. Propuestas de futuro.

Actualmente, el trabajo está en pleno desarrollo, sin embargo podemos vislumbrar algunas de las ventajas e inconvenientes que han ido apareciendo a lo largo del proceso (ver tabla 5).

Tabla 5. Ventajas y Dificultades de la Puesta en Práctica de Wikis.

\section{Ventajas}

Trabajos del alumnado con cierta calidad, al servicio del aprendizaje, no sólo de la evaluación-calificación.

El feed-back permite elaborar un trabajo guiado, supervisado y orientando a su proceso y desarrollo.

Permite la comunicación y el trabajo colaborativo entre los componentes del grupo.

Producción y publicación de las tareas al tiempo, viendo el proceso, no sólo el producto final.

Conocimiento más amplio y profundo sobre lo que se va realizando tanto en la teoría como en la práctica.

\section{Dificultades}

La propia herramienta wiki-moodle tiene muchas carencias técnicas (no poder hacer cambios en los grupos una vez iniciado el trabajo; no permite colgar imágenes, enlaces directamente en la pizarra de inicio (sí en enlaces), es un documento provisional, siempre en construcción. Impide ver una versión final-global.

No posibilita una conversación directa con el grupo (chat).

Se puede encubrir el trabajo de algunos componentes del grupo.

Exige tener internet (brecha digital). El alumnado puede creer que es una asignatura online, cuando es presencial. Mucha carga de trabajo para estudiantes y docentes.

No permite modificación de la tarea una vez realizada. 
El proceso emprendido requiere aportar soluciones a las carencias detectadas, entre las que proponemos: generar herramientas de interacción y comunicación dentro de los wikis, regular los protocolos de intervención, distinguir roles e historiales de participación, equilibrar adecuadamente la distribución de tareas en fases, distinguir las aportaciones individuales y las valoraciones del revisor, etc.

Para finalizar, indicar que tenemos la certeza de las posibilidades de los wikis para el trabajo colaborativo, aunque el profesorado implicado en la innovación ha manifestado que tiene ciertas dificultades para su utilización en la misma medida que otros instrumentos en evaluación formativa.

\section{CONCLUSIONES}

No cabe duda que los proyectos emprendidos nos permiten avanzar dando respuesta a las incertidumbres sobre la puesta en práctica de una evaluación formativa. El hecho de reflexionar juntos, dialogar sobre lo que hacemos y afrontar los obstáculos que se presentan día a día en nuestras aulas, nos enriquece como docentes al compartirlo con los demás. El trabajo en equipo y la reflexión compartida del grupo de profesorado han hecho más livianas las inseguridades que genera hacer algo diferente e innovador. Se trata de establecer un compromiso de desarrollo profesional para la mejora de la calidad en la docencia universitaria. En el proceso de innovación aquí presentado, el centro de interés de nuestra propuesta se ha basado en la metodología participativa y la evaluación formativa que se ponen en marcha en las materias de una titulación universitaria.

El uso de instrumentos alternativos a lo tradicional, como puede ser el portafolio digital y los wikis, han permitido mejorar la calidad de la participación del alumnado en las actividades individuales y grupales, generando cambios significativos en la forma de entender la enseñanza por el profesorado y el alumnado. Se necesitan cambios que debemos ir articulando progresivamente, aunque en ocasiones suponga lidiar con otras circunstancias administrativas, tales como: (a) Aumentar los horarios de tutorías. (b) Ser consciente de las tradiciones educativas y fomentar el cambio de paradigma desde la reflexión. (c) Planificar el trabajo por grupos y no de forma individual. Pero también desde el punto de vista institucional, hay factores que demandan más atención y que los hemos señalado como solución ajena al profesorado: (a) Más recursos económicos e infraestructuras. (b) Aumentar los recursos para la tutela virtual (acceso a la red, espacios y tiempos). (c) Diseñar y desarrollar tutorías colaborativas y compartidas entre el profesorado. (d) Planificar actuaciones compartidas en las que se integren los conocimientos y se hagan evaluaciones compartidas entre materias, etc.

Para terminar, parafraseando al profesor Zabalza (2003-2004), quisiéramos mostrar lo que ha significado relatar nuestra experiencia: "Ser un buen docente no tiene que significar que uno tenga que "crear ex novo" todo lo que hace en sus clases. Sería absurdo, aparte de agotador e inviable. Por eso resulta tan interesan poder contar con las aportaciones y ejemplos de otros colegas" (p. 132). 


\section{REFERENCIAS}

Anguita, R., García, S., Villagrá, S., y Jorrín, I. (2009). Wikis y aprendizaje colaborativo: lecciones aprendidas (y por aprender) en la Facultad de Educación. Red U - Revista de Docencia Universitaria. Número Monográfico V. Extraído de http://www.um.es/ead/red/M12/12-Anguita.pdf

Arter, J. A., y Spandel, V. (1992). Using Portfolios of Student Work in Instruction and Assessment. Educational Measurement: Issues and Practice, 11(1), $36-44$.

Benito, A., Cruz, A., e Icarán, E. (2005). Propuestas para afrontar las necesidades emergentes de formación del profesorado en el Espacio Europeo de Educación Superior. Revista de la RED-U, 4(2), 41-52.

Biggs, J. (2006). Calidad del aprendizaje universitario (2 $2^{\mathrm{a}}$ ed.). Madrid: Narcea.

Brown, S., y Glasner, A. (Eds.). (2007). Evaluar en la Universidad. Problemas y nuevos enfoques ( $2^{\mathrm{a}}$ ed.). Madrid: Narcea.

Buscá, F., Pintor, P., Martínez, L., y Peire, T. (2010). Sistemas y procedimientos de Evaluación Formativa en Docencia Universitaria. Resultados de 34 casos aplicados durante el curso 2007-2008. Revista de Estudios Pedagógicos, 18, 255-276.

Capllonch, M., y Castejón, F. J. (2007). La adquisición de competencias genéricas a través de una comunidad virtual de práctica y aprendizaje. Teoría de la Educación. Educación y Cultura en la Sociedad de la Información, 8(3), 168-187.

Castejón, F. J. (2009). Sistemas de evaluación formativa y mixta que utilizan la evaluación continua combinada con examen. Experiencia 1. En V. M. LópezPastor (Ed.), Evaluación formativa y compartida en Educación Superior. Propuestas, técnicas, instrumentos y experiencias (pp. 150-154). Madrid: Narcea.

Entwistle, N. (1990). Teaching and the quality of learning in higher education. En N. Entwistle (Ed.), Handbook of educational ideas and practices (pp. 103-136). London: Routledge.

González, F., y Miralbell, O. (2009). El diseño de una Wiki sobre ecoturismo como herramienta para el aprendizaje universitario de turismo en entorno virtual. Red U - Revista de Docencia Universitaria. Número Monográfico V. Extraído de http://www.um.es/ead/red/M12/14-GonzalezMiralbell.pdf

González, N. (Ed.) (2007). Desarrollo y evaluación de competencias a través del portafolio del estudiante. Santander: Universidad de Cantabria.

Hannan, A., y Silver, H. (2006). La innovación en la Enseñanza Superior. Enseñanza, aprendizaje y culturas institucionales ( $2^{\mathrm{a}} \mathrm{ed}$.). Madrid: Narcea.

Klenowski, V. (2007). Desarrollo de portafolios para el aprendizaje y la evaluación. Madrid: Narcea.

Knight, P. (2005). El profesorado de Educación Superior. Formación para la excelencia. Madrid: Narcea.

Lázaro, R., Pena, C., y Vitalaru, B. (2009). Wiki en lenguas para fines especí- 
ficos y su traducción. Red U - Revista de Docencia Universitaria. Número Monográfico $V$. Extraído de http://www.um.es/ead/red/M12/9-LazaroPenaVitalaru.pdf

López-Noguero, F. (2007). Metodología participativa en la enseñanza universitaria ( $2^{\mathrm{a}}$ ed.). Madrid: Narcea.

López-Pastor, V. M. (1999). Educación Física, Evaluación y Reforma. Segovia: Diagonal.

López-Pastor, V. M. (2004). Evaluación, calificación, credencialismo y formación inicial del profesorado: efectos y patologías generadas en la enseñanza universitaria. Revista Interuniversitaria de Formación del Profesorado, 18(3), 221-232.

López-Pastor, V. M. (2006). El papel de la evaluación Formativa en el proceso de convergencia hacia el EEES. Análisis del estado de la cuestión y presentación de un sistema de intervención. Revista Interuniversitaria de Formación del Profesorado, 20(3), 93-120.

López-Pastor, V. M. (Ed.). (2009). Evaluación formativa y compartida en Educación Superior. Propuestas, técnicas, instrumentos y experiencias. Madrid: Narcea.

López-Pastor, V. M. (2011). El papel de la evaluación formativa en la evaluación por competencias: aportaciones de la red de evaluación formativa y compartida en docencia universitaria. Red U - Revista de Docencia Universitaria, 9(1). Extraído de http://redaberta.usc.es/redu/index.php/REDU/article/ view/198.

Lyons, N. (Ed.). (2003). El uso del portafolios. Buenos Aires: Amorrortu.

Margalef, L., y Álvarez J. M. (2005). La formación del profesorado universitario para la innovación en el marco de la integración del Espacio Europeo de Educación Superior. Revista de Educación, 337, 51-70.

Martínez, L. F., Santos, M. L., y Sicilia, A. (2006). De la pedagogía del silencio a la pedagogía del diálogo. La autoevaluación y la autocalificación como formas de promoción democrática. En V.M. López-Pastor (Ed.), La evaluación en Educación Física. Revisión de los modelos tradicionales y planteamiento de una alternativa: la evaluación formativa y compartida (pp.421-444). Buenos Aires: Miño y Dávila.

Montenegro, M., y Pujol, J. (2009). Evaluación de la wiki como herramienta de trabajo colaborativo en la docencia universitaria. Red U-Revista de Docencia Universitaria. Número monográfico IV. Extraído de http://www.um.es/ead/ $\mathrm{red} / \mathrm{M} 11 /$

Pozo, M. T., y García, B. (2006). El portafolio del alumnado: una investigaciónacción en el aula. Revista de Educación, 341, 737-756.

Robles, S., Borrell, J., Fernández-Córdoba, C., Freixas, M., Pèlach, A., Pons, J.,... Sebastián, R. M. (2009). Wiki en la Universitat Autónoma de Barcelona. Red U Revista de Docencia Universitaria. Número monográfico IV. Extraído de http://www.um.es/ead/red/M11/2-Robles.pdf

Rué, J. (2007). Enseñar en la universidad. El EEES como reto para la Educación 
Superior. Madrid: Narcea.

Santos, M. L., Castejón, F. J., Díaz, M., Tejero, C., Garoz, I., y del Campo, J. (2010). Incertidumbre y desafíos ante la innovación docente en la enseñanza superior. Metodologías activas y evaluación formativa en la titulación de educación física. En $V$ Congreso Internacional y XXVI Congreso Nacional de Educación Física (formato CDrom). Barcelona: ICE Universidad de Barcelona.

Santos, M. L., Castejón, F. J., Martínez, L. F., y Muñoz, R. (2011). El portafolio digital en la plataforma Moodle como instrumento para la evaluación formativa. En EVAlfor (Ed.), EVALtrends 2011 - Evaluar para aprender en la universidad: Experiencias innovadoras en el aprendizaje a través de la evaluación (pp. 437-451). Madrid: Bubok Publishing.

Santos, M. L., Martínez, L. F., y López-Pastor, V. M. (2009) (Coord.). La innovación docente en el EEES. Experiencias de evaluación formativa y compartida en la formación inicial del profesorado. Almería: Universidad de Almería.

Zabalza, M. A. (2003-2004). Innovación en la enseñanza universitaria. Contextos Educativos, 6-7, 113-136.

Zabalza, M. A. (2007). La enseñanza universitaria. El escenario y sus protagonistas. Madrid: Narcea. 